\title{
Stimulating Cortical Drift for Non-Invasive and Efficient Treatment of a Skeletal Class III Patient
}

\author{
Teixeira CC ${ }^{a}$, Abdullah $\mathrm{F}^{\mathrm{b}}$, Sangsuwon $\mathrm{C}^{\mathrm{b}}$, Alansari $\mathrm{S}^{\mathrm{b}}$, Alikhani $\mathrm{M}^{\mathrm{b}, \mathrm{c}}$
}

a New York University, Department of Orthodontics, New York, New York

b CTOR Academy, Hoboken, New Jersey

c Harvard University, Department of Developmental Biology, Boston, Massachusetts

Corresponding Author:

Mani Alikhani

mani.alikhani@ctor.academy

Citation: Teixeira CC, Abdullah F, Sangsuwon C, Alansari S, Alikhani M. Stimulating Cortical Drift for Non-Invasive and Efficient Treatment of a Skeletal Class III Patient. Innovation. 2021. 1(1)e1. doi:10.30771/2021.1

Submitted February 15, 2021

Accepted February 26, 2021

Keywords: Neuro-Immuno-Mechanotherapy, Cortical drift, One-couple system, skeletal class III, orthopedic corrections

\begin{abstract}
Sutures and condyles are very important biological targets during orthopedic treatment of facial deformities. However, less understood is cortical drift, another natural phenomenon that if properly stimulated can play a significant role in correction of facial deformities. This phenomenon can be stimulated locally with well-planned mechanotherapy, especially free-object designs such as one-couple system. Here we present, a successful treatment of a severe skeletal Class III patient using cortical drift as an important component of a customized Neuro-Immuno-Mechanotherapy (NIM). During this non-invasive treatment approach, significant correction of the Class III jaw relation was achieved through maxillary advancement without surgery or extractions. The patient did not report any side effects or discomfort related to this treatment. This case study demonstrates the importance of using innovative approaches combining proper stimulation of the biological response with a customized mechanotherapy for treatment of severe craniofacial deformities.
\end{abstract}




\section{Background}

A severe Class III skeletal problem can be the result of a maxillary deficiency or mandibular protrusion, or a combination of both. Due to interference between upper and lower anterior teeth, a significant posterior open bite is usually observed when these patients are guided to the point of interference. As a result of this interference these patients advance their lower jaw to accommodate a posterior occlusion, which gradually worsens their skeletal deformity. Advancement of the mandible can also decrease their anterior facial height due to overclosure of the mandible, and prevent proper eruption of posterior teeth and posterior vertical growth of the jaws.

Traditionally, orthognathic surgery was considered the treatment of choice for patients with severe Class III skeletal problems [1]. However, this approach is not without side effects [2]. In addition, this type of treatment usually is postponed until patient finishes growing, which can affect the patient's quality of life as a child and teenager. Due to these limitations many parents seek non-surgical treatments.

Another option for treating skeletal Class III malocclusions is camouflage where some teeth are extracted to compensate for the skeletal disharmony [3]. This approach does not address the underlying skeletal problem, often leading to compromised esthetic outcomes, making this option unpopular among parents.

A more recent option that is gaining popularity is applying orthopedic forces to surgical plates [4] placed in the maxilla. This option can improve the result of orthopedic treatment, more so in adults than children, in whom the presence of permanent tooth buds and insufficient bone density are contraindications for using surgical plates. In addition, these procedures require surgical intervention to place and remove the surgical plates, which makes many parents uncomfortable.

While classical orthopedic treatments focus on applying static force to separate circum-maxillary sutures to displace the maxilla forward or prevent condylar growth [5], these approaches have demonstrated limited success and unpredictable results. Therefore, a non-surgical treatment for skeletal Class III malocclusion remains elusive.

In light of new biological discoveries on the mechanism of sutural growth, advances in understanding the role of the central nervous system (CNS) in coordinating jaw growth, and the latest research in bone biology showing cortical drift as one of the main determinants in the final shape of the jaws, here we propose a new orthopedic approach that targets these different biological processes, called Neuro-Immuno-Mechanotherapy (NIM).

In this case report we present a young patient with a severe Class III skeletal pattern that positively responded to a new mechanotherapy regimen targeting maxillary sutures and cortical drift. This patient was too young for a surgical treatment option, therefore we proposed to use NIM therapy to improve the outcome of his orthopedic treatment. A personalized mechanotherapy plan was developed to address all the patient's problems non-surgically.

\section{Patient Presentation, Etiology and Diagnosis}

A healthy 11.5-year-old male patient presented to the clinics of CTOR Academy with a chief concern of an "underbite." The patient was visiting his dentist frequently and oral hygiene was good with no cavities or any signs of periodontal problems. A temporomandibular joint exam did not reveal any temporomandibular joint signs and patient did not report any symptoms.

When asked to slowly close his mouth and stop at first contact, the patient showed an edge-to-edge relation of the anterior teeth. In this bite position, the frontal portrait photograph revealed facial symmetry and lip competence, while the lateral portrait photograph showed a concave profile (Figure 1). The nasolabial angle and the chin-throat angle were acceptable (92.6o, 126o, respectively) and the labiomental angle was obtuse (161.8o). The upper lip was deficient and lower lip was protruded relative to the E-line (-3.3 $\mathrm{mm}, 2.7 \mathrm{~mm}$, respectively) (Table I). Upon smiling he exhibited a straight smile line, 100\% incisal display, and increased buccal corridor width.

The intra-oral exam, performed with the patient holding his bite at first occlusal contact, showed an anterior crossbite with -1 to -3 $\mathrm{mm}$ overjet and $0-10 \%$ overbite. His maxillary dental midline was $2 \mathrm{~mm}$ to the right of his facial midline; however, the mandibular dental midline was coincident to the facial midline. Both right and left molars and canines presented a Class III occlusal relation; however, the Class III dental relation was more severe in the left side. With the mandible in this position, the patient demonstrated a posterior open bite that extended from the second molar to the central incisors. The patient had an ectopically erupted maxillary right canine, which was elevated and displaced buccally, as well as a lingually displaced maxillary right lateral incisor. In the mandible, the patient presented moderate crowding. The patient's maxillary right second molar was not erupted, the maxillary left second molar was partially erupted, and the mandibular second molars were erupting. All frenum attachments were within normal limits. 

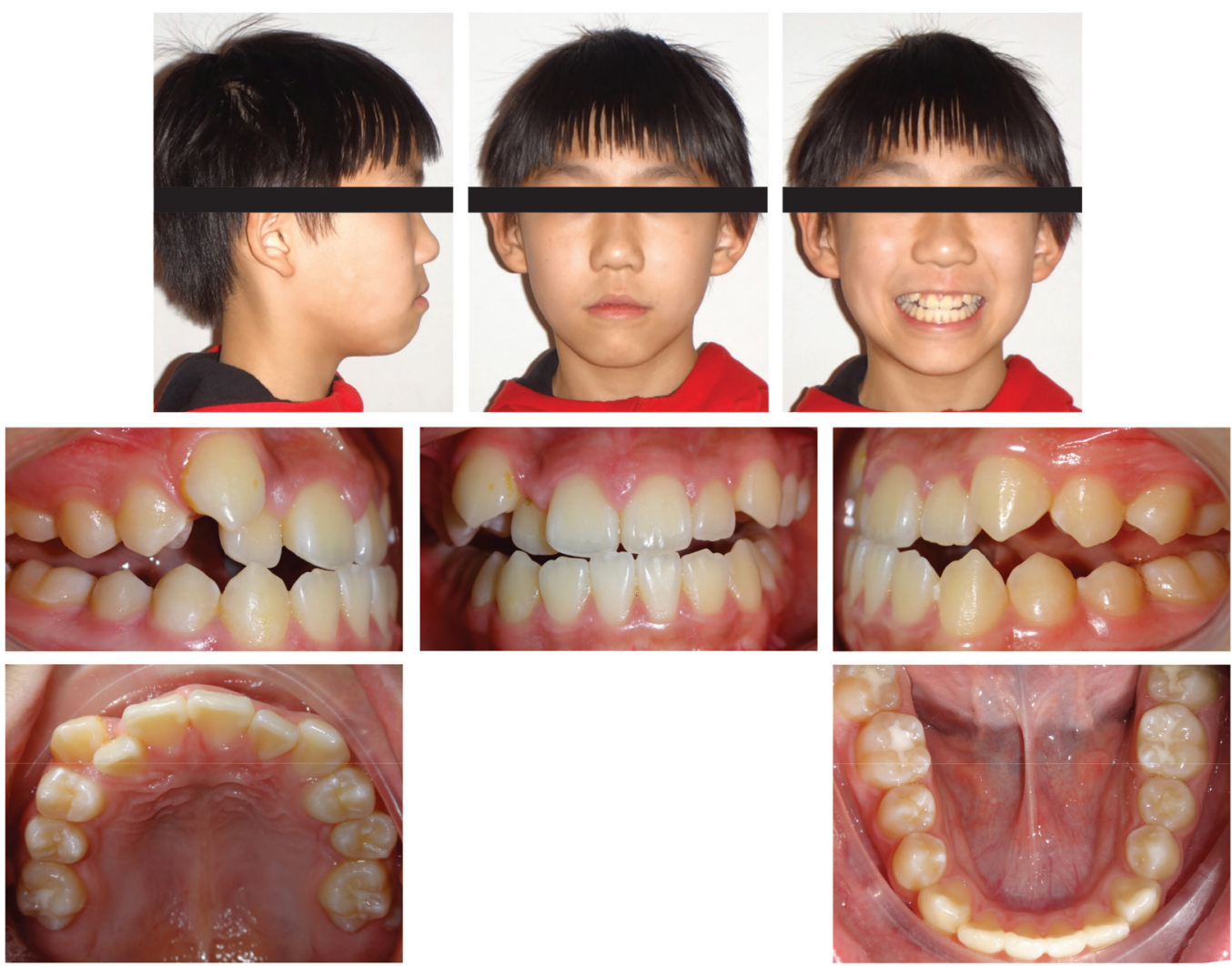

Figure 1: Pre-treatment portrait and intra-oral photographs. Pre-treatment portrait and intraoral photographs show the patient's occlusion when asked to slowly close his mandible until the first tooth touches. In this position, the lateral profile photograph shows a concave profile with the lower lip projecting more anteriorly than the upper lip. Frontal portrait photographs show lip competency at rest and 100\% incisal display while smiling. Intraoral photographs reveal the dental interference that causes a functional shift upon closing, a constricted maxilla, anterior crossbite, severe crowding in the maxillary arch, mild crowding in the mandibular arch and the ectopic position of the maxillary right lateral incisor and canine. The maxillary right second molar was not present in the arch, but the maxillary left second molar was partially erupted, and the mandibular second molars were erupting in their correct positions.
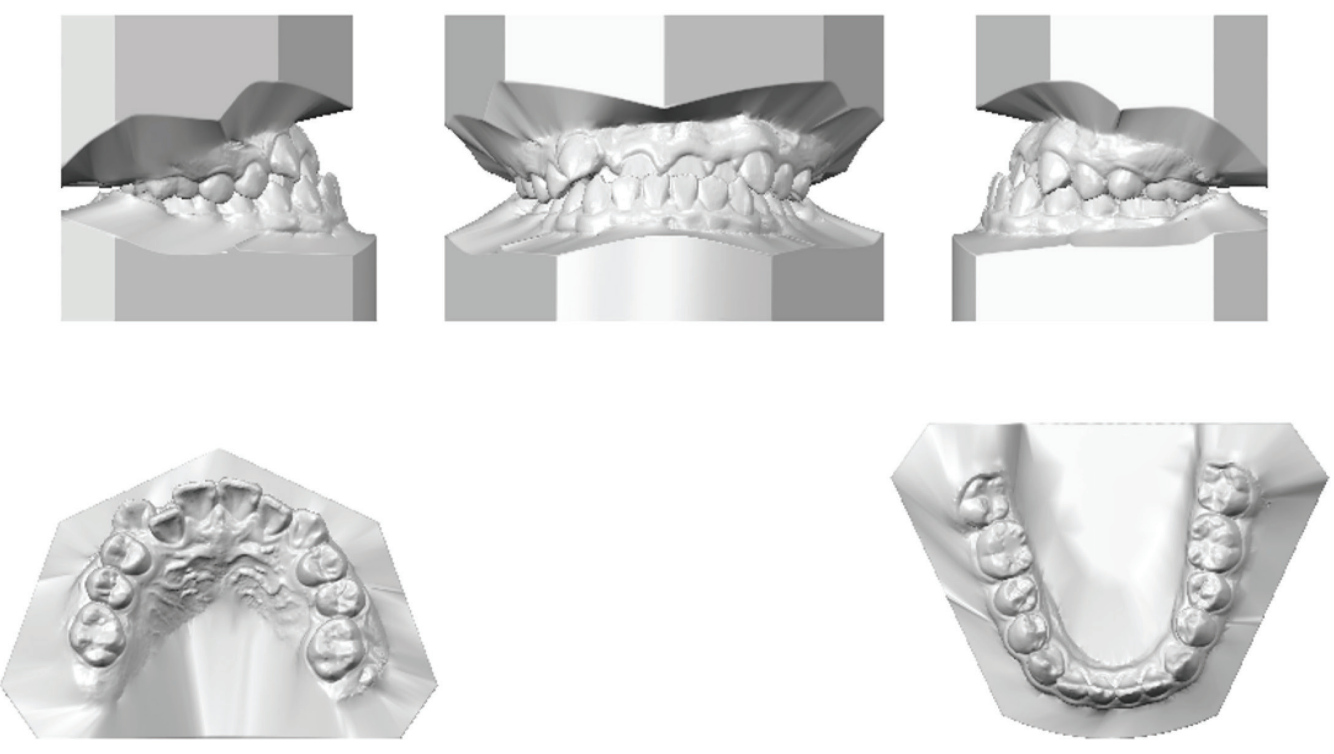

Figure 2: Pre-treatment digital casts. Pre-treatment digital casts show the patient's occlusal relation when the mandible shifts forward allowing posterior teeth to occlude. Cast analysis shows a Class III canine and molar occlusal relation, an anterior crossbite on the maxillary incisors, a negative overjet (-2.6 mm) and overbite (5.1 mm). Severe maxillary crowding and mild mandibular crowding were observed, with the maxillary right lateral incisor and canine blocked out of the arch. The maxillary inter-canine and inter-molar widths were $38.2 \mathrm{~mm}, 51.5 \mathrm{~mm}$, respectively, and the mandibular inter-canine and inter-molar widths were $27.5 \mathrm{~mm}, 43.9 \mathrm{~mm}$, respectively. The patient's maxillary right second molar was not present in the arch, while the maxillary left second molar was partially erupted and the mandibular second molars were erupting. 


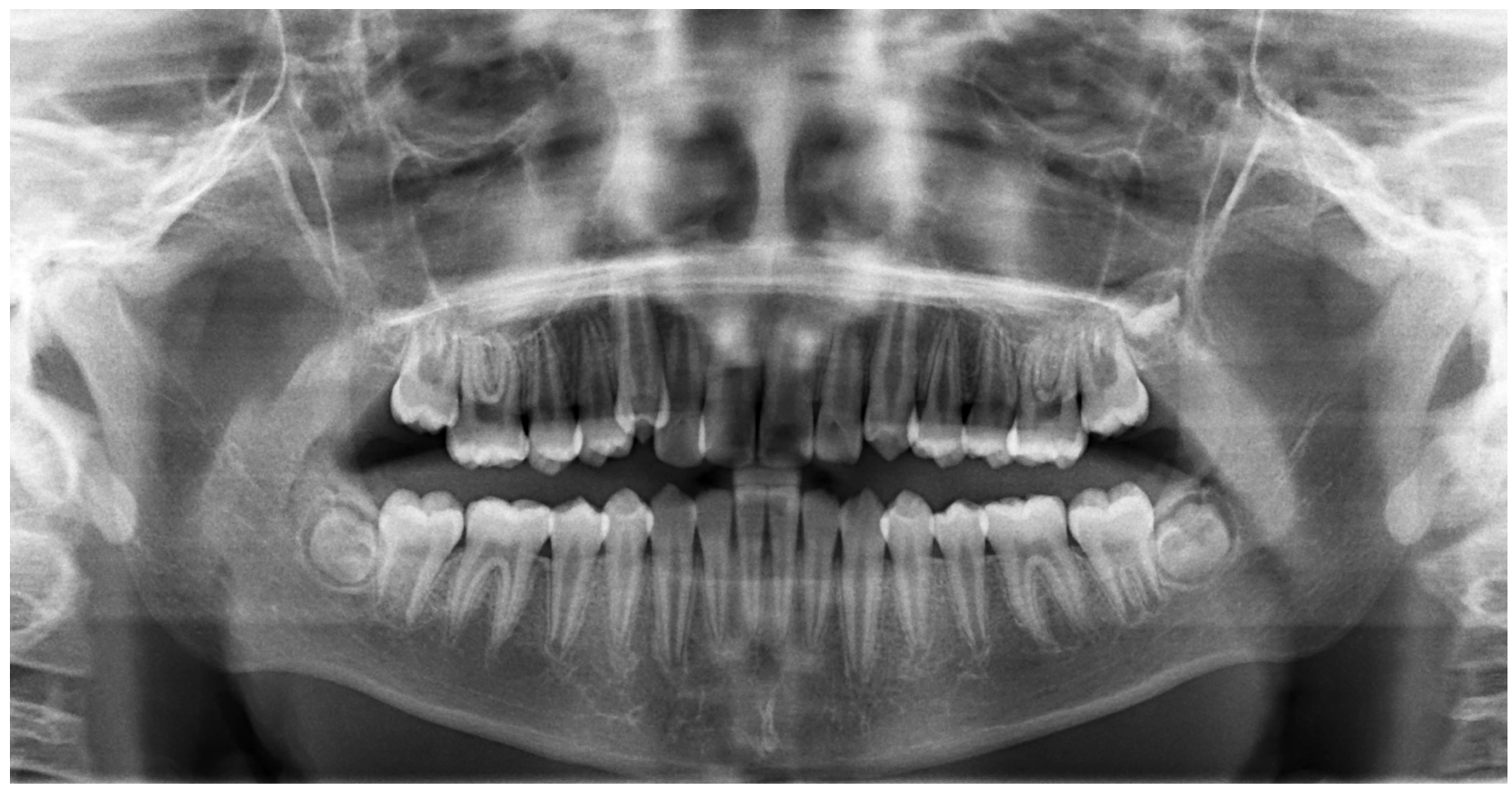

Figure 3: Pre-treatment panoramic radiograph. Panoramic radiograph shows development of a complete dentition except for the absence of the upper right third molar tooth bud. The apices of several teeth were not fully formed, while first molars and incisors displayed spindle-like roots. An asymmetry of the left and right condylar neck lengths was noted.
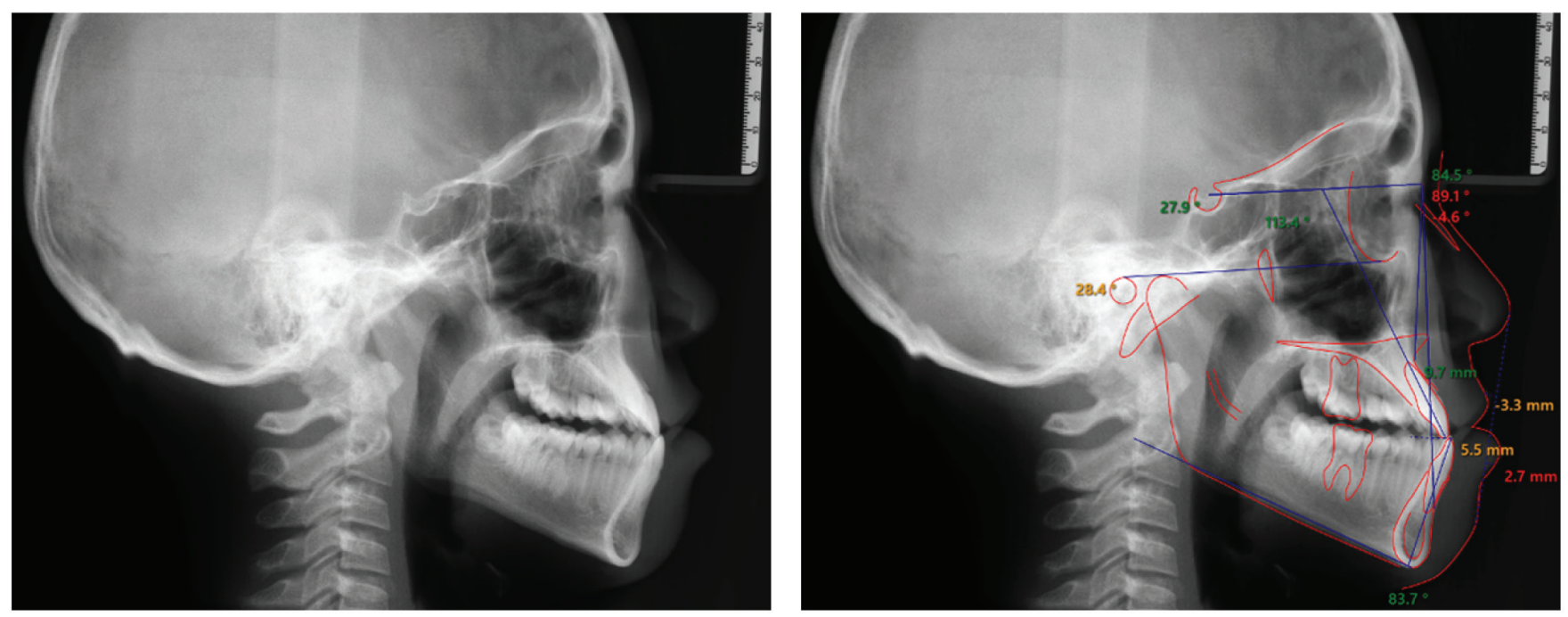

Figure 4: Pre-treatment lateral cephalometric radiograph and analysis. Cephalometric radiograph was taken with the patient posturing the mandible backwards into the first tooth contact position. Cephalometric analysis showed a hypodivergent mandible angle, a Class III skeletal relation, anterior crossbite with proclined maxillary incisors and normal inclination of the mandibular incisors. 


\begin{tabular}{|lccc|}
\hline \multicolumn{1}{|c}{ Measurement } & Initial Value & Final Value & Norm \\
\hline SNA Angle & $84.5^{\circ}$ & $88.6^{\circ}$ & $80.7 \pm 3.4^{\circ}$ \\
\hline SNB Angle & $89.1^{\circ}$ & $88^{\circ}$ & $77.3^{\circ} \pm 3.1^{\circ}$ \\
\hline ANB Angle & $-4.6^{\circ}$ & $0.6^{\circ}$ & $3.4 \pm 2^{\circ}$ \\
\hline FMA & $28.4^{\circ}$ & $29.7^{\circ}$ & $27.7 \pm 5.8^{\circ}$ \\
\hline SN-MP & $27.9^{\circ}$ & $28.8^{\circ}$ & $33.2 \pm 5.1^{\circ}$ \\
\hline Upper Incisor Angle with SN & $113.4^{\circ}$ & $117.3^{\circ}$ & $102.4 \pm 5.5^{\circ}$ \\
\hline IMPA & $83.7^{\circ}$ & $84.8^{\circ}$ & $94.8 \pm 7.2^{\circ}$ \\
\hline Upper Incisor to NA mm & $9.7 \mathrm{~mm}$ & $7.2 \mathrm{~mm}$ & $4.3 \pm 2.8 \mathrm{~mm}$ \\
\hline Lower Incisor to NA mm & $5.5 \mathrm{~mm}$ & $5.7 \mathrm{~mm}$ & $4.9 \pm 2.8 \mathrm{~mm}$ \\
\hline Upper Lip to E-plane & $-3.3 \mathrm{~mm}$ & $-0.5 \mathrm{~mm}$ & $-2 \pm 2 \mathrm{~mm}$ \\
\hline Lower Lip to E-plane & $2.7 \mathrm{~mm}$ & $1 \mathrm{~mm}$ & $-2 \pm 2 \mathrm{~mm}$ \\
\hline
\end{tabular}

Table 1: Cephalometric Analysis of Pre- and Post-treatment lateral chephalograms. Angular and distance measurements were completed between craniofacial skeletal and dental landmarks identified on pre- and post-treatment lateral cephalograms $\left({ }^{\circ}\right.$ - degrees, $\mathrm{mm}$ - milimeters )

During full-intercuspation as displayed in the cast analysis (Figure 2), we observed full Class III molar and canine relationships on both sides, an anterior crossbite with -2 to $-4 \mathrm{~mm}$ overjet and $40 \%-60 \%$ overbite. No posterior open bite was observed in this mandibular position, but when the patient displayed a mandibular functional shift to the left, a $5 \mathrm{~mm}$ midline discrepancy appeared.

Cast analysis showed the maxillary inter-canine and inter-molar widths were $38.2 \mathrm{~mm}$ and $51.5 \mathrm{~mm}$, respectively, and the mandibular inter-canine and inter-molar widths were $27.5 \mathrm{~mm}, 43.9 \mathrm{~mm}$, respectively.

The panoramic radiograph (Figure 3) revealed a complete dentition with the apices of many teeth still forming, consistent with the patient's chronological age. A condylar asymmetry was observed with the condylar neck in the right side longer than condylar neck in the left side.

The lateral cephalometric radiograph (Figure 4) was taken when the patient was in the edge-to-edge incisal relation and the mandible was not postured forward. The lateral cephalometric analysis (Table 1) showed a Class III skeletal relationship (ANB $\left.=-4.6^{\circ}\right)$ with a hypo-divergent profile $\left(\mathrm{FMA}=28.4^{\circ}, \mathrm{SN}-\mathrm{MP}=27.9^{\circ}\right)$. The maxillary incisor inclination was increased $\left(\mathrm{U} 1^{\circ}-\mathrm{SN}=\right.$ $\left.113.4^{\circ}\right)$, while the mandibular incisor inclination was decreased $\left(\operatorname{IMPA}=83.7^{\circ}\right)$.

\section{Treatment Objective}

The overall treatment objective was to correct the skeletal discrepancies, establish a stable functional occlusion and improve the facial and dental esthetics. To achieve this overall objective, we planned the following specific treatment objectives for the face, skeleton and dentition:

I. Facial objectives included improving the facial profile, the lip position relative to the E-line, decreasing the buccal corridors, and maintaining the mandibular profile.

II. Skeletal objectives included correcting the maxillary transverse deficiency, and the sagittal disharmony by protraction of the maxilla to achieve Class I skeletal relationship.

III. Dental objectives included expanding the maxillary arch and developing the mandibular arch to correct the anterior crossbite, resolving the crowding, protracting the upper dental arch, correcting the maxillary dental midline, eliminating the posterior open bite, obtaining proper overjet and overbite relation; establishing a Class I canine and molar relationship; and improving the gingival height of contour around the anterior teeth. 


\section{Treatment Options}

Different treatment options were presented and discussed with the parents. Parents and patient opposed any surgical intervention following completion of patient's growth, tooth extractions or usage of any type of temporary anchorage devices, but they were open to any non-invasive approach to increase the envelop of correction, enhance the biological response and improve the orthopedic treatment outcome. A customized NIM therapy plan was proposed.

To stimulate the biphasic stages of sutural stimulation [6], a special maxillary expander was designed to not only deliver transverse forces to the hemimaxillae but also to produce labial forces on the anterior teeth. The maxillary expander design included hooks for applying anterior displacement forces through a face mask. This was combined with Alikhani Bite Block therapy to deprogram the CNS. Cortical drift was stimulated by a one-couple system that directed maxillary alveolar bone movement downward and forward. After the new position of the maxilla was achieved, free-object segmental mechanics followed by a full fixed appliances set up were used to establish the final occlusion. Fixed lingual retainers were bonded on upper and lower anterior teeth and special removable retainers were designed to not only hold the treatment results, but also to actively provide positive signals to the CNS.

\section{Treatment Outcome}

Total treatment time was 28 months, including few months interruption during Covid-19 2020 Pandemic. All treatment objectives were achieved by a customized NIM plan:

\section{Facial Changes (Figure 5):}

A) Profile analysis showed improvement in the upper and lower lip position relative to the E-line.

B) Smile analysis showed a wider maxilla and a significant decrease of the buccal corridor width. Smile esthetics were also significantly improved.

\section{Skeletal corrections and lateral cephalometric analysis (Figure 8):}

A) The post-treatment lateral cephalometric analysis (Table 1; Figure 8) showed that the Class III skeletal jaw relation was corrected by significant advancement of the maxilla (SNA increased from 84.5 to 88.6).

B) Patient did not show any major changes in mandibular plane angle.

C) Superimposition of the radiographs taken before and after treatment (Figure 9) showed that the maxillary and mandibular incisors extruded, and all first molars were extruded and uprighted.

D) Superimposition of mandibular canal shows vertical growth of the condyles.

E) Soft tissue analysis indicated that the distance of the upper and lower lips to the E-line was improved (-0.5 mm, $1 \mathrm{~mm}$, respectively).

\section{Dental corrections (Figures 5 and 6):}

A) Maxillary dentition was expanded significantly (inter-molar width increased by $4.1 \mathrm{~mm}$, while inter-canine width increased by $1.9 \mathrm{~mm})$.

B) Proper overjet (2 $\mathrm{mm})$ and overbite (40\%) were established.

C) Crowding in both maxillary and mandibular arches was resolved.

D) Both maxillary and mandibular dental midlines were aligned with the facial midline.

E) Class I canine and molar occlusion was established.

F) Gingival esthetic analysis showed that the gingival height of contour was leveled around the anterior teeth.

G) Post-treatment panoramic radiograph (Figure 7) showed good root alignment with mild and localized blunting of dental roots. 

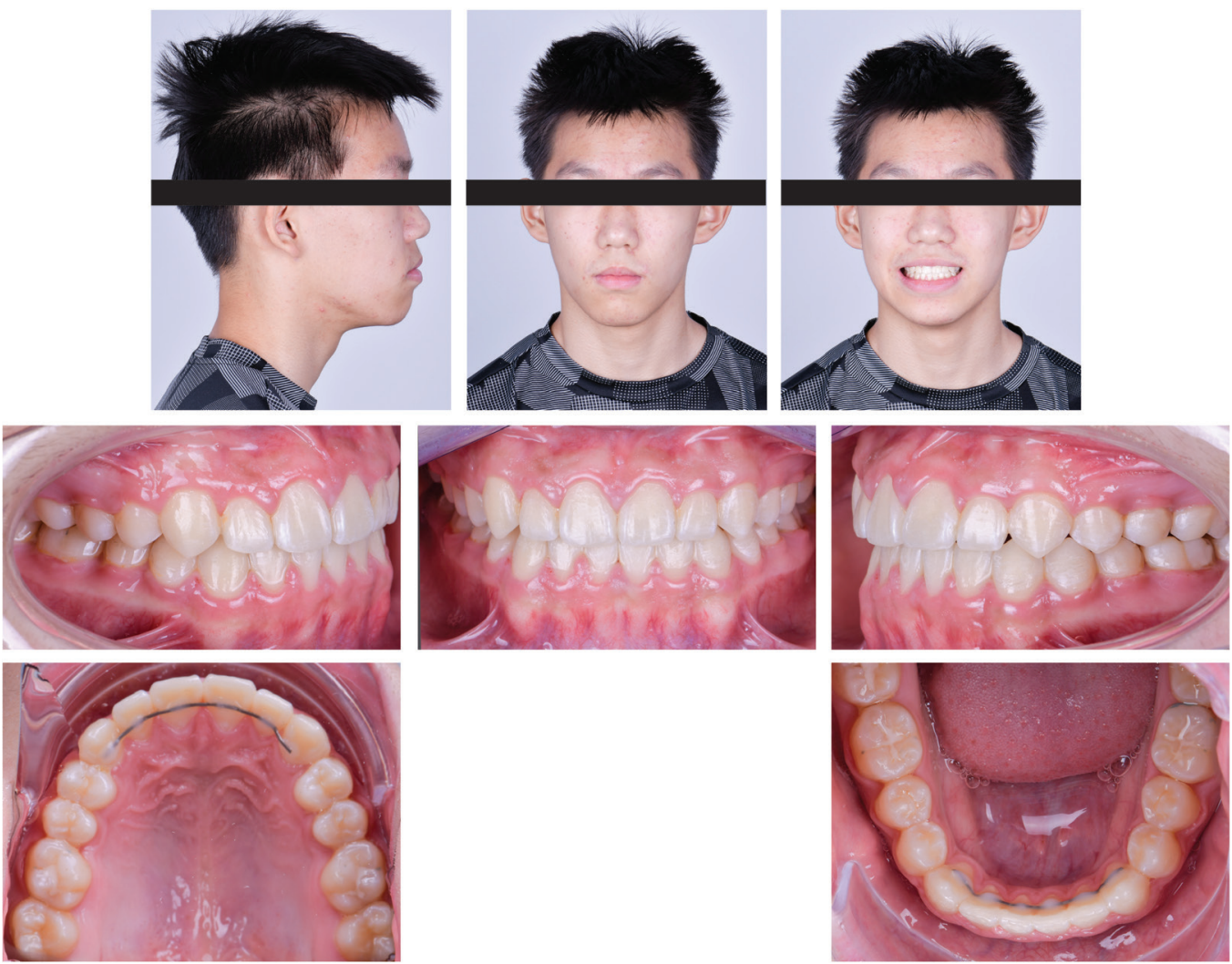

Figure 5: Post-treatment portrait and intra-oral photographs. Post-treatment photographs reveal a well-balanced straight facial profile, with relaxed lip posture and improved smile esthetics. Intra-oral photographs showed expansion of the maxillary arch, development of the mandibular arch, coinciding midlines, a Class I molar and canine occlusion, the correction of the anterior crossbite into an ideal overjet and overbite relation. Both maxillary and mandibular dental midlines were aligned with the facial midline. Gingival margins were leveled and the gingival height of contour improved around the anterior teeth. Total treatment time was 28 months.
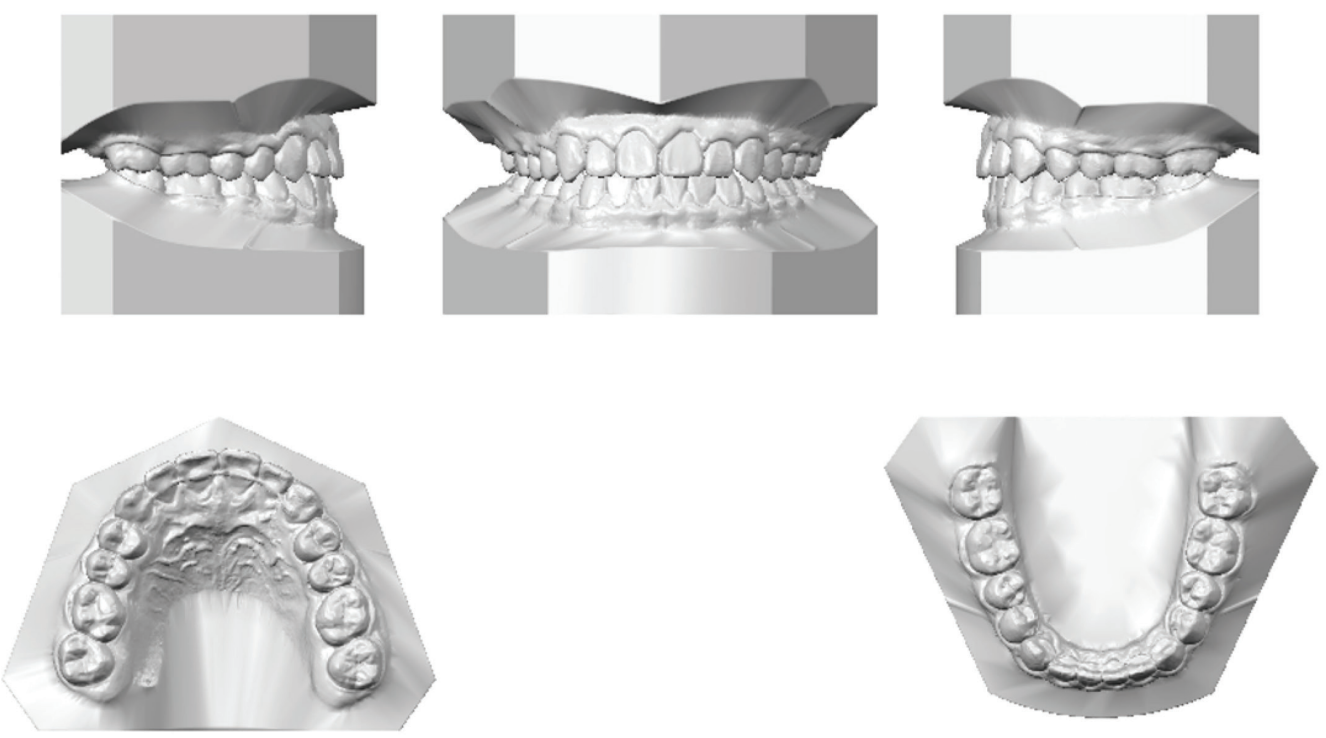

Figure 6: Post-treatment digital casts. Post-treatment digital cast analysis showed increased maxillary arch dimensions (intermolar width increased by $4.1 \mathrm{~mm}$, while inter-canine width increased by $1.9 \mathrm{~mm}$ ), coincident dental midlines, Class I molar and canine occlusal relationship, ideal overiet $(2 \mathrm{~mm})$ and overbite $(1.7 \mathrm{~mm})$. 


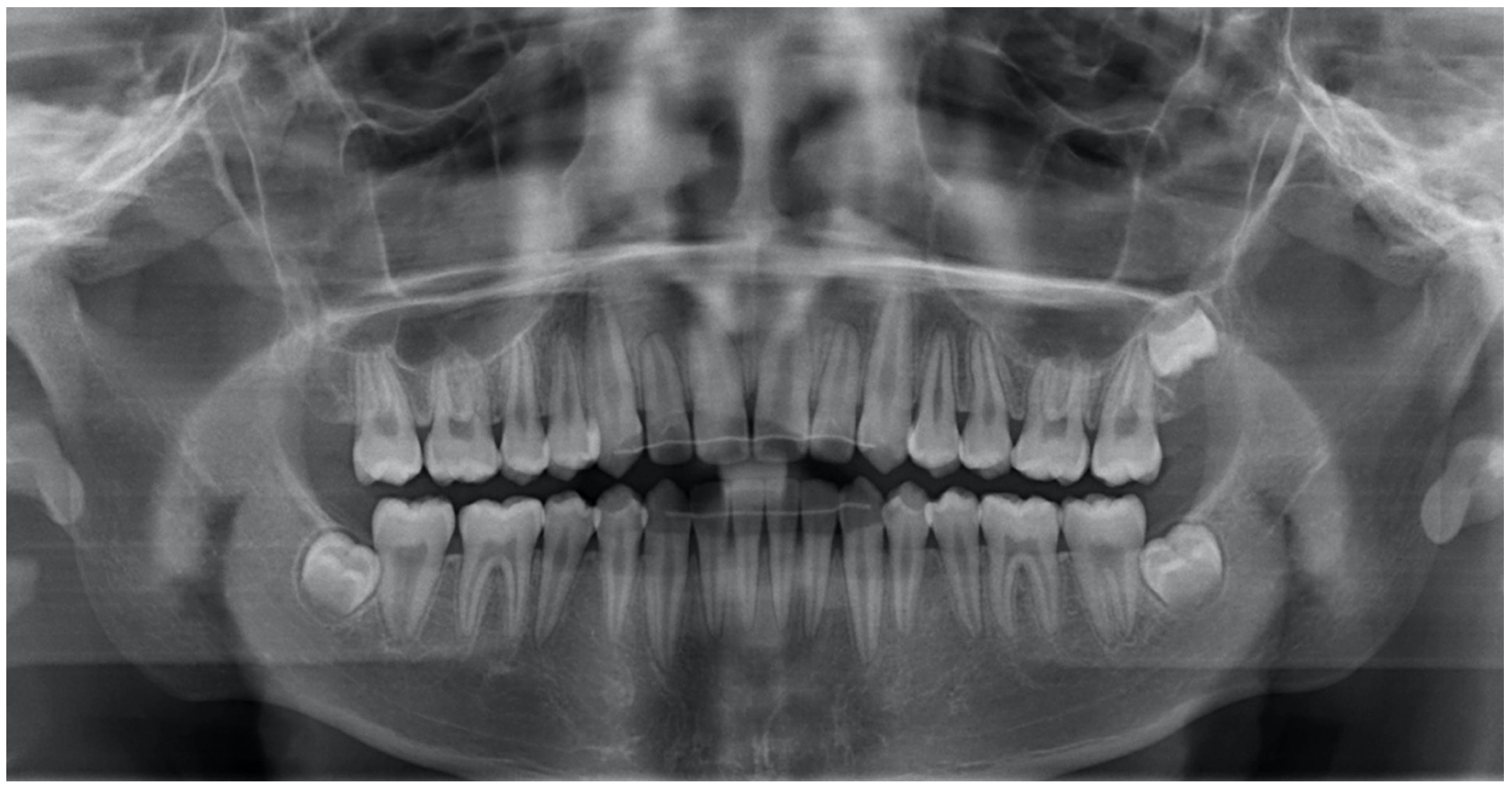

Figure 7: Post-treatment panoramic radiograph. Panoramic radiograph at the end of treatment showed good root parallelism, relative blunting of the roots of the maxillary laterals, presence of third molar tooth buds except for the maxillary right third molar tooth bud, and improved condylar symmetry.
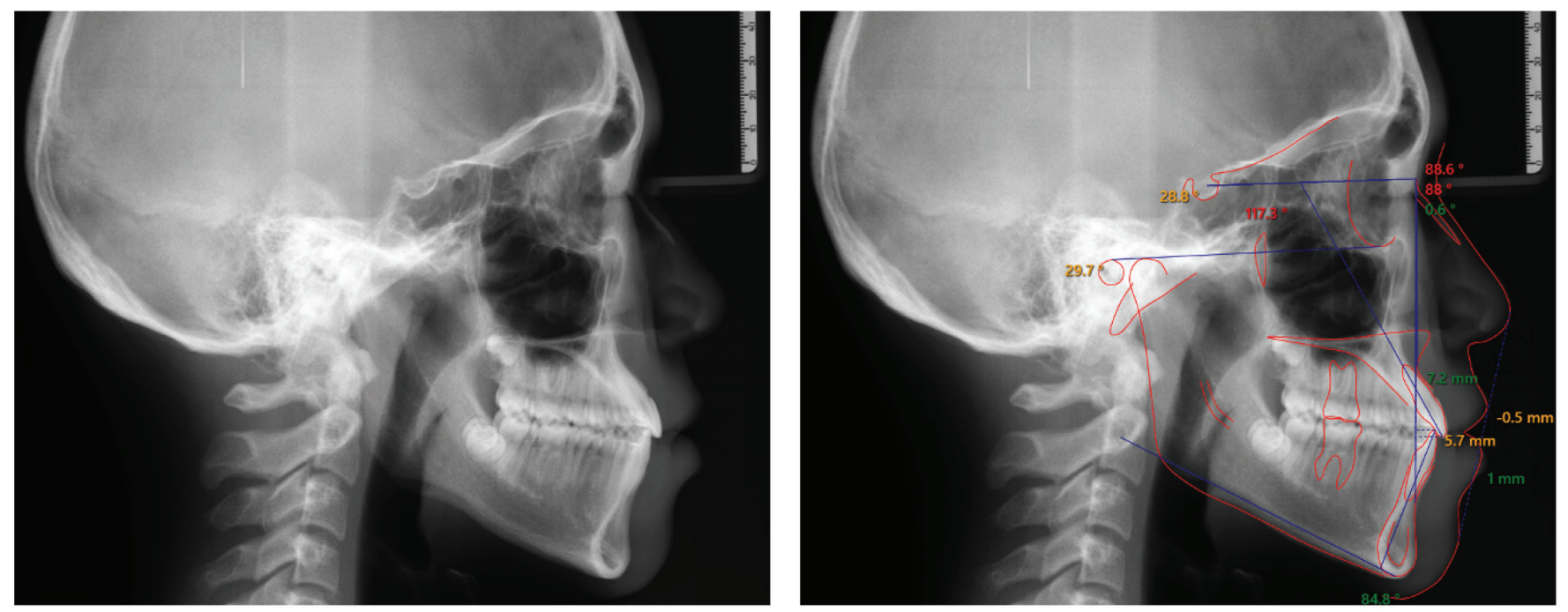

Figure 8: Post-treatment lateral cephalometric radiograph and analysis. Post-treatment cephalometric analysis shows overall improvement in the relation between maxillary and mandibular dental arches, correction of anterior crossbite into ideal overjet, ideal overbite, and improved soft tissue profile and lip position. 


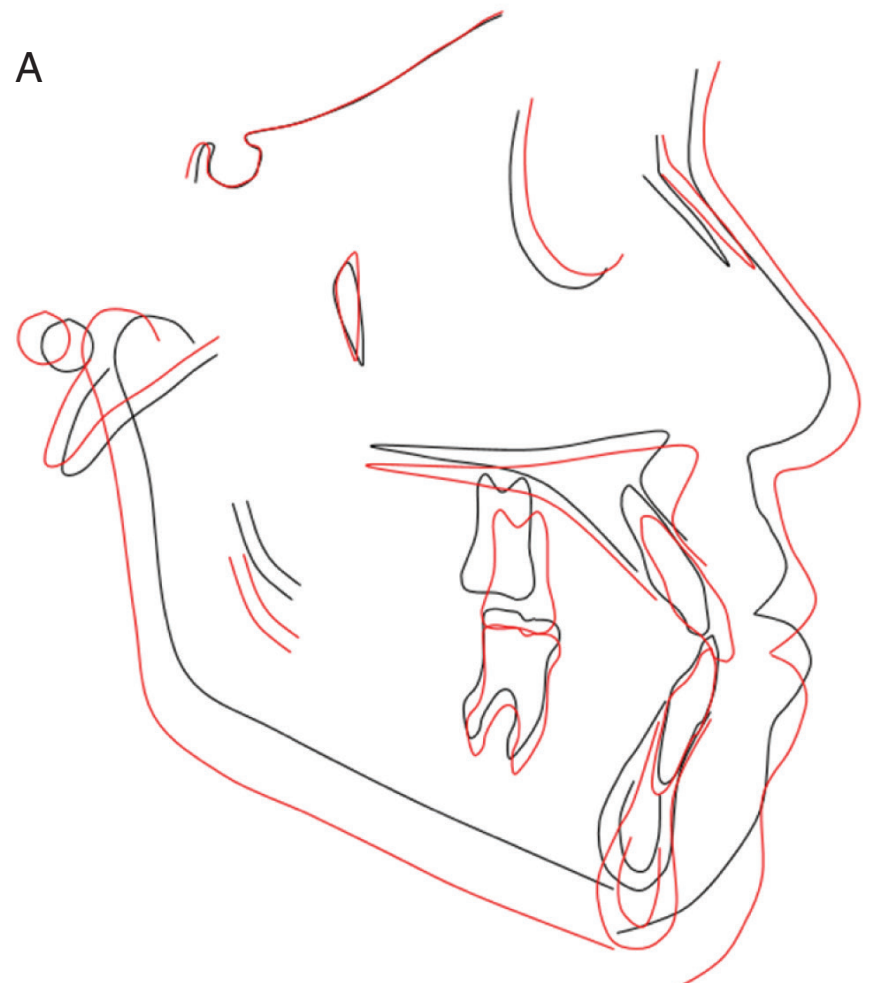

B
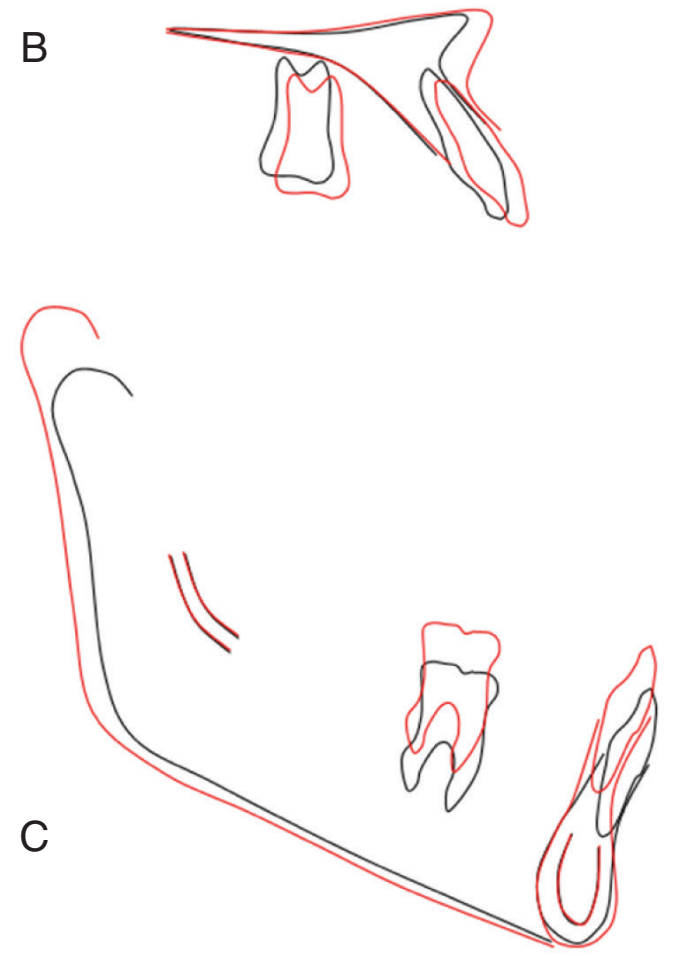

Figure 9: Superimposition of pre- and post- treatment cephalometric tracings. Cephalometric superimposition of pre-treatment (black tracing) and post-treatment (red tracing) on the anterior cranial-base shows significant vertical maxilla and mandible growth, and significant maxillary advancement resulting in a Class I jaw relation, proclination of maxillary incisors, and extrusion of both maxillary anterior and posterior teeth (A). Superimposition based on the body of the maxilla, showed molar extrusion and uprighting and incisor extrusion with proclination (B). Superimposition based on the inferior-alveolar nerve and inner profile of the mandibular symphysis revealed incisor extrusion without significant change in inclination, molar extrusion and uprighting, and vertical growth of the mandible and condyle (C).

\section{Discussion}

Previously we have shown that to achieve adequate maxillary expansion and advancement, cytokines should be activated in the circum-maxillary sutures, followed with monocyte recruitment and their activation into osteoclasts [6]. Osteoclast activity at the sutures allows the maxilla to move freely in response to orthopedic forces. Osteoclast activity is followed by coordinated osteoblast activation to reestablish the integrity of the skeleton with the maxilla in its new position.

Activating the bone remodeling machinery not only impacts the sutures, but also stimulate cortical drift, which plays a significant role in determining maxillary shape and size. Cortical drift is a coordinated activity of osteoclasts and osteoblasts that gradually displace different components of the bone in space. This phenomena occurs naturally during life however, stimulating localized cortical drift can be a purposeful and versatile tool in reshaping maxilla or mandible to correct skeletal discrepancies. Cortical drift, in this case, was directed by applying a one-couple system that guided the alveolar bone growth in the anterior maxillary area [7].

According to CNS Theory of growth [8], the CNS plays a significant role in controlling the form of the craniofacial skeleton. The use of Alikhani Bite Block therapy during treatment aided in re-establishing proper CNS signaling to the craniofacial complex. Without re-establishing CNS signaling the results of orthopedic treatment may not be stable, which likely accounts for relapse following traditional orthopedic approaches. Gradual correction of CNS signaling using Alikhani Bite Block therapy during treatment and later during retention stabilizes the result of orthopedic treatment [8].

The use of a free-object design to address the majority of the patient's problems, enabled us to maximize the movement of different targets and minimize unwanted side effects. Placing brackets on all the teeth from the beginning of treatment and the use of a continuous archwire would jeopardize the treatment and create unpredictable results. Similarly, the use of clear aligners would not address the biological requirement for the efficient treatment of these cases.

This case study demonstrates the importance of incorporating biological advances and customizing the mechanotherapy plan to meet the patient's specific needs. Based on this case report, cortical drift is an important biological target and one of the main components of NIM therapy approach which offers a non-invasive, efficient and cost-effective treatment option for correcting severe skeletal malocclusions. 


\section{Applied Innovation}

Orthopedic treatment mostly focuses on sutures and condyles as biological targets. Here we demonstrated that cortical drift as important biological target during treatment of craniofacial deformities. By localized stimulation of this biological phenomenon through application of carefully planned mechanotherapy it is possible to extend the boundaries of orthopedic corrections. However, stimulating cortical drift is only one aspect of the innovative approach developed by CTOR (Consortium for Translational Orthodontics Research) called NIM therapy. In this approach by establishing a proper CNS signaling along with a personalized mechanotherapy it is possible to return the normal function and insure a successful outcome of orthopedic treatment.

\section{References}

1. Busby BR, Bailey LJ, Proffit WR, Phillips C, White RP, Jr. Long-term stability of surgical class III treatment: a study of 5-year postsurgical results. Int J Adult Orthodon Orthognath Surg. 2002;17(3):159-70. Epub 2002/10/02. PubMed PMID: 12353934.

2. Patel PK, Morris DE, Gassman A. Complications of orthognathic surgery. J Craniofac Surg. 2007:18(4):975-85; Quiz 86-8. Epub 2007/08/02. doi: 10.1097/ scs.0b013e318068442c. PubMed PMID: 17667699.

3. Mora DR, Oberti G, Ealo M, Baccetti T. Camouflage of moderate Class III malocclusions with extraction of lower second molars and mandibular cervical headgear. Prog Orthod. 2007:8(2):300-7. Epub 2007/11/22. PubMed PMID: 18030376.

4. De Clerck H, Cevidanes L, Baccetti T. Dentofacial effects of bone-anchored maxillary protraction: a controlled study of consecutively treated Class III patients. Am J Orthod Dentofacial Orthop. 2010;138(5):577-81. Epub 2010/11/09. doi: 10.1016/j.ajodo.2009.10.037. PubMed PMID: 21055597; PubMed Central PMCID: PMCPMC3033914.

5. Major PW, elBadrawy HE. Maxillary protraction for early orthopedic correction of skeletal Class III malocclusion. Pediatr Dent. 1993;15(3):203-7. Epub 1993/05/01. PubMed PMID: 8378159.

6. Alikhani M, Alansari S, Al Jearah MM, Gadhavi N, Hamidaddin MA, Shembesh FA, et al. Biphasic sutural response is key to palatal expansion. Journal of the World Federation of Orthodontists. 2019;8(1):9-17. doi: https://doi.org/10.1016/j.ejwf.2019.01.002.

7. Alikhani M. Clinical Guide to Accelerated Orthodontics, With a Focus on Micro-Osteoperforations: Springer International Publishing: 2017.

8. Alikhani M. Mechanotherapy in Orthodonitcs Volume III: In Press, CTOR Press, Hoboken NJ. 